\title{
A Road Extraction Method Based on Region Growing and Mathematical Morphology from Remote Sensing Images
}

\author{
Yunhe Liu' ${ }^{*}$, Chi $\mathrm{Ma}^{2}$, Li Li², Xiaoyan Xing2, Yong Zhang², Zhigang Wang², Jiuwei Xu² \\ ${ }^{1}$ Chang Guang Satellite Technology Co., Ltd., Changchun, China \\ ${ }^{2}$ Department of Transportation of Jilin Province, Changchun, China \\ Email: *lyh2426@163.com
}

How to cite this paper: Liu, Y.H., Ma, C., Li, L., Xing, X.Y., Zhang, Y., Wang, Z.G. and $\mathrm{Xu}$, J.W. (2018) A Road Extraction Method Based on Region Growing and Mathematical Morphology from Remote Sensing Images. Journal of Computer and Communications, 6, 91-97.

https://doi.org/10.4236/jcc.2018.611008

Received: August 28, 2018

Accepted: November 12, 2018

Published: November 19, 2018

\begin{abstract}
Road traffic is the important driving factor for economic and social development. With the rapid increase of vehicle population, road traffic problems such as traffic jam and traffic accident have become the bottleneck which restricts economic development. In recent years, natural disasters frequently occur in China. Therefore, it is essential to extract road information to compute the degree of road damage for traffic emergency management. A road extraction method based on region growing and mathematical morphology from remote sensing images is proposed in this paper. According to the road features, the remote sensing image is preprocessed to separate road regions from non-road regions preliminarily. After image thresholding, region growing algorithm is used to extract connected regions. Then we sort connected regions by area to exclude the small regions which are probably non-road objects. Finally, the mathematical morphology algorithm is used to fill the holes inside the road regions. The experimental results show that the method proposed can effectively extract roads from remote sensing images. This research also has broad prospects in dealing with traffic emergency management by the government.
\end{abstract}

\section{Keywords}

Region Growing, Mathematical Morphology, Road Extraction, Remote Sensing Images

\section{Introduction}

There are lots of information in remote sensing images, and the rapid development of remote sensing technology has gradually made it one of the main means 
of acquiring geographic information data. With the appearance and application of high-resolution remote sensing images, more information can be extracted from remote sensing images. But at the same time, more details in high-resolution images make it more difficult to extract information.

Road extraction from remote sensing images is one of the hot spots and difficult problems, and has been widely researched by domestic and foreign scholars [1] [2] [3] [4]. According to the degree of automation of existing road extraction methods, road extraction technology is generally divided into semi-automatic road extraction and automatic road extraction. Semi-automatic road extraction is accomplished by means of human-machine interaction. The main idea is to manually provide some prior information such as the initial seed point of the road. Then the computer will judge and identify the road automatically. The semi-automatic methods include: based on dynamic programming methods, based on template matching methods, based on active contour model methods and so on. Automatic road extraction interprets and identifies roads by extracting and understanding road image features. That is, the features of the road are analyzed first, and then a certain type of road is identified automatically by means of artificial intelligence, computer vision, pattern recognition and other methods. The automatic methods include: based on parallel lines methods, knowledge-based methods, based on statistical model methods and so on. Baumgartner and Steger [5] proposed an automatic road extraction method by using of several versions of the same aerial image with different resolutions. Senthilnath and Rajeshwari [6] proposed a road extraction based on texture progressive analysis. The methods based on mathematical morphology [7] [8] could eliminate the noise. The method based on object-oriented [9] constructs the knowledge base for extracting multi-dimensional feature space. Most of road extraction methods are based on high-quality image information. That is, the less noise the image contains, the more abundant the road information extracted is.

\section{Method}

Although there are many objects in the remote sensing image such as buildings, roads, trees and so on, the roads still have some features that can be distinguished from other non-road objects. Generally, roads have the following features:

1) The gray scale in the road changes little.

2) The road and its adjacent region differ in color.

3) The width of the road varies little and the length is much greater than the width.

4) Most of the roads in the image are the parallel roads or the cross roads.

Thinking about the road features mentioned above, we design a suitable method to extract road objects. Because the road region changes little in color and differs from its adjacent region, we could distinguish the road region by its color. 
Gray stretching based gray histogram could enhance the grayscale of road region. After image thresholding, we use region growing algorithm to extract connected regions. Because the roads change little in width and are always crossing, we could consider that the pixel number in road regions are relatively numerous. In other word, the small regions are generally non-roads such as houses, ponds, trucks and so on. Therefore, we could sort the connected regions by size to exclude the small ones. There are vehicles on the roads, and the color of vehicles and roads differ. It may cause that holes appear in the road region in the binary image. The closing algorithm is used to fill holes. Finally, we merge extraction result with original image and get the final image.

\subsection{Preprocessing}

\section{Gray Stretching}

The method that the piecewise linear function can be used to enhance the contrast of image is called gray stretching. In fact, it increases the grayscale of regions of interest and decreases the grayscale of other regions. The advantage of piecewise linear transformation is that it has various forms and can be synthesized arbitrarily, while the disadvantage is that it requires more pre-defined parameters. The function formula of piecewise linear transformation is as follows:

$$
f(x)=\left\{\begin{array}{lc}
\frac{y_{1}}{x_{1}} x & x<x_{1} \\
\frac{y_{2}-y_{1}}{x_{2}-x_{1}}\left(x-x_{1}\right)+y_{1} & x_{1}<x<x_{2} \\
\frac{255-y_{2}}{255-x_{2}}\left(x-x_{2}\right)+y_{2} & x>x_{2}
\end{array}\right.
$$

The most important parameters in the formula are $\left[x_{1}, x_{2}\right]$ and $\left[y_{1}, y_{2}\right]$. According to the algorithm description, parameters $\left[x_{1}, x_{2}\right]$ determine the range of gray scale needed to be converted, and parameters $\left[y_{1}, y_{2}\right]$ determine the slope of linear transformation. Gray stretching can control the distribution of output grayscale histogram flexibly. In this paper, we set parameters as:

$$
x_{1}=40, x_{2}=150, y_{1}=20, y_{2}=230
$$

Figure 1 shows the result of image preprocessing.

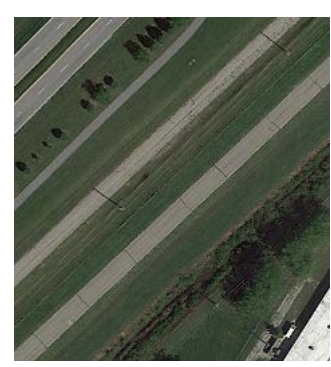

(a)

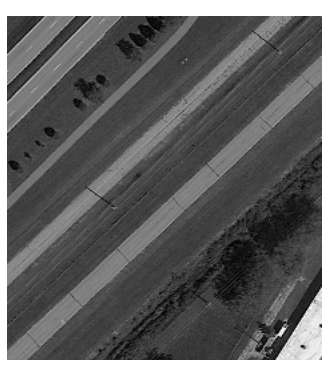

(b)

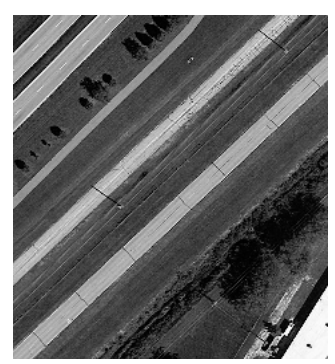

(c)

Figure 1. Processing results of image preprocessing. (a) Original image; (b) Gray image; (c) Processing result of gray stretching. 


\subsection{Road Extraction}

\subsubsection{Region Growing}

The basic idea of region growing is to assemble pixels with similar property to form new regions. First, a seed pixel is found as the starting point for each region to be divided, and then the pixels with similar properties to seed pixel around the seed pixel are merged into the region where the seed pixel is located. Continue the process of treating these new pixels as new seed pixels until no more pixels which meet the condition can be found. The region growing algorithm has been widely used because of its fast speed and pertinence. In remote sensing images, compared with the road regions, the non-road objects such as the houses at the side the road are usually distributed in isolation. Considering the above characteristic, we use to region growingto exclude the small non-road regions. Figure 2 shows the result of image region growing. We can see that the numerous small regions in Figure 2(a) disappear in Figure 2(b).

\subsubsection{Mathematical Morphology}

Mathematical morphology is an image analysis method based on strict mathematical theory which is a science of quantitative description of geometric structures by the method of set theory. The basic idea of mathematical morphology is to use the pre-defined structural elements (such as disks and squares) to detect information and shapes in images. The choose of structural elements has a great impact on the results, and the processing results with structural elements of different shapes and sizes are dramatically different. Therefore, suitable structural elements should be selected according to the processing objects. At present, mathematical morphology is widely used in image processing for noise removal, feature extraction, edge detection, image segmentation, texture analysis, image compression and reconstruction. The main operations of mathematical morphology include erosion and dilation, opening and closing. The followings are introduced one by one:

- Erosion and dilation operations

Erosion and dilation operations are the basic operations of mathematical morphology and are also a set of dual operations. Erosion operation is used to detect image with a structural element to find areas within the image where the structural element can be placed. Erosion operation can eliminate the small components in the image and remove the noise. The erosion operation of structural elements $\mathrm{B}$ on image $\mathrm{A}$ is expressed as $A \ominus B$, which is defined as:

$$
A \ominus B=\{X: B+X \subset A\}
$$

Dilation operation uses structural elements to fill the small holes and the hollows at the edges, so it can be defined as the complementary set of erosion operation. The dilation operation of structural elements $\mathrm{B}$ on image $\mathrm{A}$ is expressed as $A \oplus B$, which is defined as:

$$
A \oplus B=\left\{A^{c} \ominus(-B)\right\}^{c}
$$

- Opening and closing operations 
Opening and closing operations are combined operations based on erosion and dilation operations, which are also duality relations. The opening operation is the result of erosion first and dilation later. It could remove the salt and pepper noise and smooth the inner edge, which achieves the effect of low-pass filtering. The closing operation is the result of dilation first and erosion later. It could full the small holes in the interior of the object and connect the smooth boundaries, which achieves the effect of high-pass filtering.

In general, there are many shadows of trees and poles in the road. Therefore, we can see that lots of holes appear in Figure 2(b). In this paper, closing operation is used to fill the holes inside the road regions, which is shown in Figure 2 (c).

\subsection{Experiment}

The experimental data is an aerial color image. The road shows a high brightness and similar gray level connected network in the image, as shown in Figure 3(a). First, the original image is preprocessed. By comparison Figure 3(b) and Figure $3(c)$, we can find the grayscale of road regions are enhanced by gray stretching. After image thresholding by OSTU, there are many noises and small regions of non-roads in binary image, as shown in Figure 3(d). Though we can remove the

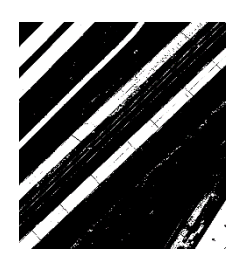

(a)

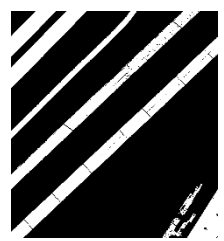

(b)

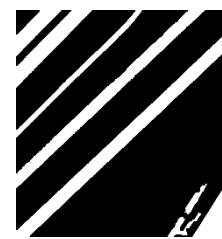

(c)

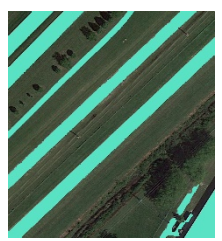

(d)

Figure 2. Processing results of road extraction. (a) Binary image; (b) Processing of region growing; (c) Processing result of; (d) Processing result of to exclude the small regions closing operation road extracion.

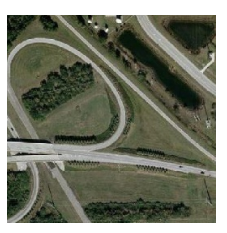

(a)

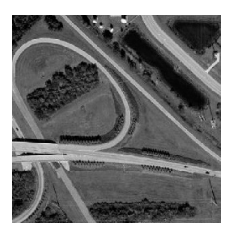

(b)

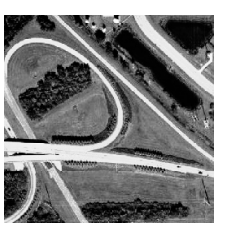

(c)

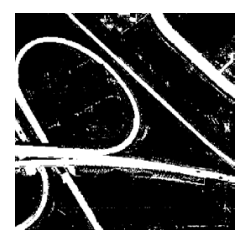

(d)

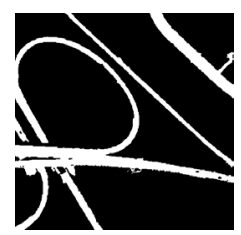

(e)

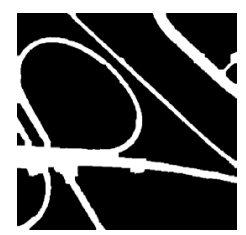

(f)

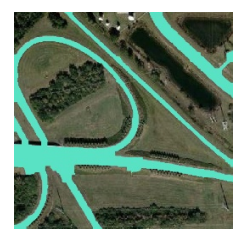

(g)

Figure 3. Processing results of road extraction. (a) Original image; (b) Gray image; (c) Processing result of gray stretching; (d) Binary image; (e) Processing of region growing; (f) Processing result of; (g) Processing result of to exclude the small regions closing operation road extracion. 
noises by filtering in some way, the non-road regions cannot be removed by filtering. By processing of region growing, we could get many regions which varies a lot in size. Figure 3(e) is the results of excluding the small regions. Based on this, the closing operation is used to fill the holes inside the roads. It must be noted that the size of structural element determines the precision of result. However, due to the complex and changeable geometric features of road in remote sensing images, the structural elements are not universal. It is necessary to select appropriate structural elements according to the actual situation to achieve the best results.

\section{Conclusion}

According to the unique features of road objects in high resolution remote sensing image, an automatic road extraction method based on region growing and mathematical morphology is proposed in this paper. First, the image is pretreated using gray stretching to separate road regions from non-road regions preliminarily. After image thresholding, region growing algorithm is used to extract connected regions and exclude the small pieces of non-road regions. The mathematical morphology algorithm is used to improve the precision of extraction result. According to the two experimental results, this method can not only effectively extract road objects, but also remove the vehicles objects in the roads. In the meanwhile, this method can exclude the ground objects which have the similar color to road objects. This method has a poor performance on road segments with shadow effects. These problems need to be further studied.

\section{Conflicts of Interest}

The authors declare no conflicts of interest regarding the publication of this paper.

\section{References}

[1] Lin, Z. and Liu, Z. (2003) Methods and Prospects of Road and Linear Structure Extraction from Remote Sensing Image. Geomatics and Information Science of $\mathrm{Wu}$ han University, 1, 90-93.

[2] Ye, F., Su, L., Li, S. and Tang, J. (2006) Review and Thought of Road Extraction from High Resolution Remote Sensing Images. Remote Sensing for Land \& Resources, 1, 12-17.

[3] Wang, P., Wang, L., Fen, X., et al. (2009) Review of Road Extraction from Remote Sensing Images. Remote Sensing Technology \& Application, 24, 284-290.

[4] Xiang, H. (2013) Review and Prospect of Road Feature Extraction from High Resolution Remote Sensing Images. Geomatics \& Spatial Information Technology, 36, 202-206.

[5] Baumgartner, A., Steger, C., Mayer, H., et al. (1999) Automaticroad Extraction Based on Multi-Scale. Grouping, and Context. Pe \& Rs, 65, 777-785.

[6] Senthilnath, J., Rajeshwari, M. and Omkar, S.N. (2009) Automatic Road Extraction Using High Resolution Satellite Image Based on Texture Progressive Analysis and Normalized Cut Method. Journal of the Indian Society of Remote Sensing, 37, 
351-361. https://doi.org/10.1007/s12524-009-0043-5

[7] Zhu, C., Wang, Y., et al. (2004) Road Extraction from High-Resolution Remotely Sensed Image Based on Morphological Segmentation. Acta Geodaetica et Cartographica Sinica, 33, 347-351.

[8] Li, L., Liu, J. and Yin, Z. (2005) Road Extraction from High Resolution Remote Sensing Image Based on Mathematic Morphology. Remote Sensing Information, 20, 9-11.

[9] Tang, W., Zhao, S. and Wang, P. (2008) Object-Oriented Road Information Extraction from High Resolution Imagery. Geo-Information Science, 10, 257-262. 